\title{
Iris Feature Extraction and Recognition based on Gray Level Co-occurrence Matrix (GLCM) Technique
}

\author{
Rabab A. Rasool \\ Collage of Engineering \\ Material Department \\ Baghdad, Iraq
}

\begin{abstract}
Biometric features have received great attention for many applications. Iris recognition is one of the most modern biometric technique that is used for accurate and reliable authentication. Recently, Gray-Level Cooccurrence Matrix (GLCM) is one of the advanced techniques used for features extraction. In this paper, an iris recognition system proposed involves; preprocessing, feature extraction, and matching processes. After the preprocessing process, the feature extraction technique based on GLCM has been applied to pure iris region to extract features. Only one of the second-order statistical features known as contrast will be calculated from the generated co-occurrence matrix and stored it as a numerical feature vector in CASIA-v4.0-iris database. During recognition, the matching metric based on Euclidean distance has been used for authentication. Results have demonstrated $(99.5 \%)$ highly accuracy rate with (0.02) FAR, and (0.01) FRR.
\end{abstract}

\section{General Terms}

Iris Recognition Technology and application of Iris Recognition Technology (authentication and security).

\section{Keywords}

Gray Level Co-occurrence Matrix (GLCM), Feature extraction, Euclidean distance, Iris recognition system.

\section{INTRODUCTION}

In recent year, biometric features have received great attention for many applications based on features obtained from one's face [1], finger [2], voice [3] and/or iris [4]. Iris recognition system is widely used in terms of recognition and identification performance [5] owing to a random texture of iris is stable over one's lifetime. Feature extraction is a serious work to extract significant features from iris images. Various algorithms have been adopted by researchers for feature extraction which are based on different transform. A little research has been recorded using statistical techniques for iris recognition [6].

Recently, Gray-Level Cooccurrence Matrix (GLCM) is one of the advanced techniques used for texture feature extraction was proposed by Haralick et al. back in 1973 [7]. It shows how often a pixel value is known as the reference pixel with the intensity value $i$ occurs in a specific relationship to a pixel value known as the neighbor pixel with the intensity value $j$. So, each element $(\mathrm{i}, \mathrm{j})$ of the matrix is the number of occurrences of the pair of a pixel with value $i$ and a pixel with value $\mathrm{j}$ which are at a distance $d$ relative to each other. The spatial relationship between two neighboring pixels can be specified in many ways with different offsets and angles, four possible spatial relationships $(0 ; 45 ; 90$ and 135). Figure 1 illustrates the details of the process to generate four symmetrical co-occurrence matrices considering a $4 \times 4$ image represented with one neighboring pixel $(d=1)$ along four possible directions as $\left\{\left[\begin{array}{ll}0 & 1\end{array}\right]\right.$ for 0 ; [-1 1$]$ for $45 ;[-10]$ for 90 and $[-1-1]$ for 135$\}$. Each element of the GLCM is the number of times that two pixels with gray ton $i$ and $j$ are the neighborhood in distance $\mathrm{d}$ and direction $\theta$ [8].

Many statistical features introduced by Haralick et al are generated by calculating the co-occurrence matrix to detect image texture properties indirectly as it contains considerable image information such as contrast, homogeneity, energy, entropy, etc.

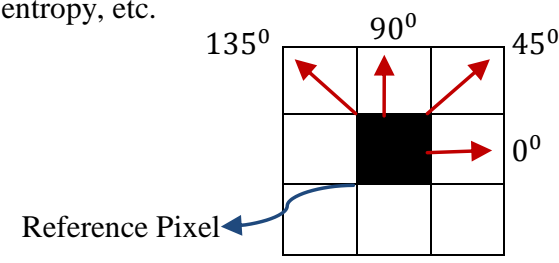

Fig 1: Co-occurrence matrix directions for extracting texture features

In this paper, a statistical feature extraction approach based on GLCM is proposed and implemented to extract desired iris features. Firstly, after capturing two eye images from the CASIA V4.0 database [9], the preprocessing is applied. It divides into two main processes; segmentation and normalization. The normalized iris image is enhanced. After normalization, feature extraction is applied to the cooccurrence matrix and create an iris feature vector. And finally, the Euclidean distance is used for matching purpose. The system tested on total 100 eye images for 10 persons. Each person has 10 images one for testing and the other for training. Database structure includes 10 classes for 10 persons and each class has 9 images for each person. The system performance is evaluated to obtain an accuracy rate, false acceptance rate (FAR), and the false rejection rate(FRR).

\section{IMAGE PREPROCESSING}

The original image needs to be pre-processed to remove irrelevant parts such as the eyelid, eyelash, pupil, etc. The preprocessing is consisting of two processes: iris segmentation and iris normalization.

\subsection{Iris Segmentation}

Segmentation process indicates to isolate pure iris region by creating inner and outer rings which allow to minimizing iris region noises caused by reflection, pupil, and eyelids or eyelashes to obtain a clean iris image for extraction and comparison. In this work, iris segmentation is carried out using a Hough transform technique with canny edge detection for detecting the iris and pupil boundaries. The proposed system has been tested only on the successfully segmented iris images. Iris and pupil boundaries detection shown in Figure 2. 


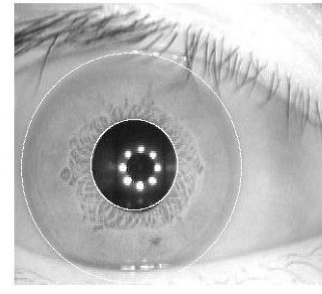

Fig 2: Iris and pupil boundaries detection

\subsection{Iris Normalization}

Iris Normalization is a process of converting the Iris from circular region to rectangular region by using Dugman's rubber sheet model to normalize the iris segmented image. Iris region is obtained as a normalized part with respect to iris boundaries and center of the pupil. The rubber sheet model in Figure 3 remaps points within the iris region to a pair of polar coordinates $(r, \theta)$ where $r$ is on the interval $[0,1]$ and $\theta$ is angle $[0,2 \pi]$. Iris normalization is shown as in Figure 4.

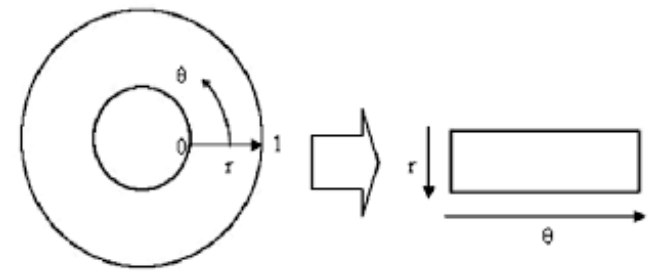

Fig 3: Daugman's rubber sheet model

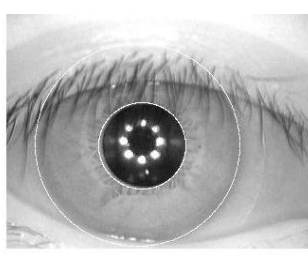

(a)

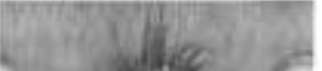

(b)
Fig 4: Iris normalization. (a) Iris segmented image. (b) Iris normalized image

\section{IRIS FEATURE EXTRACTION}

Feature Extraction plays a basic role in the iris recognition system. This paper is focusing on the feature extraction technique, while most of the iris recognin systems implementation is employed the binary representation in the iris feature extraction, in this work, the gray scale representation based on the gray level co-occurrence matrix (GLCM) technique is used and the normalized image is not converted into binary form.

The proposed GLCM algorithm works by forming a " $5 \times 5$ " detection window on each testing and training normalized iris images that scroll over them. This selected window has an appropriate size to make storing and retrieving processes more efficient. The algorithm will be depending on the orientation angle to describe an iris image through GLCM. It starts in the top left corner with angle " 0 ". After calculating the cooccurrence matrices, only one of the second-order statistical features known as "contrast" will be computed indirectly based on these generated matrices. This statistic measures the difference between the highest and the lowest values of a contiguous set of pixels as shown in (1)[8].

$$
\text { Contrast }=\sum_{i, j=1}^{k} P(i, j)(i-j)^{2}
$$

Where, " $\mathrm{K}$ " array dimension, and ' $\mathrm{i}$ ', $\mathrm{j}$ ' are the iterators on the Contrast array.

From the generated "Contrast" matrix only the diagonal values are selected because its principal diagonal is related to homogeneous regions and the nonzero elements far from it represent high contrast occurrences [8] and stored as numerical feature vector (VC) in the database given by (2).

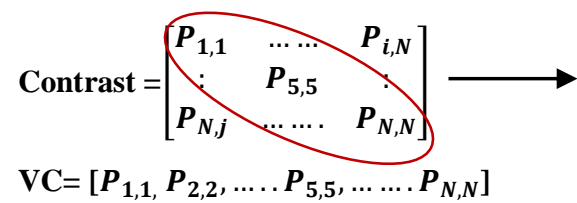

\section{MATCHING AND RECOGNITION}

Euclidean distance (ED) will be used to get the matching decision. It is the most commonly used distance. Euclidean distance computes the square root of the sum of the squares of the difference between vectors. The ED is computed between the test iris feature vector $(\mathrm{Vt})$ and stored vectors $(\mathrm{VC})$ given by (3).

$$
\mathbf{E D}=\sqrt{\sum(\boldsymbol{V t}-\boldsymbol{V C})^{2}}
$$

The evaluated values (ED) are stored in the distance array (Dist) given by (4). Each row represents a distance vector for each class.

$$
\operatorname{Dist}=\left[\begin{array}{ccc}
E D_{i, j} & \ldots \ldots & E D_{i, N} \\
: & \ddots & : \\
E D_{M, j} & \ldots \ldots & E D_{M, N}
\end{array}\right]
$$

Where,

$\mathrm{M}$ is the total number of classes in the database,

$\mathrm{N}$ is the total number of iris region images in each class, 'i', 'j' are the iterators on the Dist array.

Then, the minimum values (Min) of a matching distance of each row (class) are found out from Dist array and stored them in one vector (Min_Dist) given by (5).

$$
\operatorname{Min} \_D i s t=\left[\operatorname{Min}_{1}, \operatorname{Min}_{2}, \ldots \ldots \ldots . \operatorname{Min}_{M}\right]
$$

Finally, for recognition, the minimum value from the generated vector above is chosen as a matching score. This score is compared with a matching threshold that authenticates the user as genuine or imposter. A matching threshold for this system is selected where FAR and FRR are equal, or where FRR is minimum. In this paper, the optimum matching threshold chosen was 0.25 value. FAR and FRR are defined in (6), (7) [10].

$$
\begin{aligned}
\text { FAR } & =\frac{\text { Wrongly accepted indivitual }}{\text { Total number of wrong matching }} \\
\text { FAR } & =\frac{\text { Wrongly rejected indivitual }}{\text { Total number of correct matching }}
\end{aligned}
$$

The two iris images will be classified as the same class if the matching score is less than the matching threshold. So, the user is considered as a genuine, otherwise, these two iris images will be classified as different classes then the user is considered as an imposter.

Experimental results of this proposed system give an accuracy rate of $99.5 \%$, FAR of 0.02 , and FRR of 0.01 . These are very good results compared with other results of other iris recognition system methods in other researches. Comparison of the obtained accuracy has been made with other existing 
methods of feature extraction in iris recognition system. Table 1 is shown as the result of this comparison.

Table 1. Experimental results comparison

\begin{tabular}{|c|c|}
\hline Methods & $\begin{array}{c}\text { Accuracy } \\
\text { Rate \% }\end{array}$ \\
\hline J.Daugman & $100 \%$ \\
\hline Wildes [11] & $99.8 \%$ \\
\hline Charles O Ukpai [12] & $98.8 \%$ \\
\hline Ankush Kumar [13] & $97.5 \%$ \\
\hline The proposed method & $99.5 \%$ \\
\hline
\end{tabular}

\section{CONCLUSION}

In this work, a second-order statistical measurement was based use the GLCM technique to extract iris features. The contrast metric was extracted to recognize the human identity using Euclidean distance metric based on generating numerical feature vectors which are stored in the CASIA v4.0 database. In the proposed approach is tested on total 100 eye images for 10 persons and each person has 10 images. Results have done demonstrated $(99.5 \%$ ) highly accuracy rate that approximate or better other recognition systems with a recorded 0.02 FAR and 0.01 FRR at relatively long execution time. In the result, a reliability of the Iris recognition system is favorable. For the future work, it can be evaluated of more iris features, which moreover helps to improve the accuracy of the system and can be tested on a large number of CASIA v4.0 database iris images or on other databases, to improve the working of the system on real-time applications.

\section{ACKNOWLEDGMENTS}

Exceptional thanks to Prof. Muthana H. H. who have contributed towards support and encourage this work.

\section{REFERENCES}

[1] Giot, R., Hemery, B., and Rosenberger, C. 2010 Low cost and usable multimodal biometric system based on keystroke dynamics and 2-D face recognition. In: Proceedings of twentieth IEEE international conference on pattern recognition, pp. 1128-1131, 23-26 August 2010.

[2] Cao, K., Eryun, L., and Jain, A., K., "Segmentation and enhancement of latent fingerprints: A coarse to fine ridge structure dictionary", IEEE Trans. Pattern Anal. Mach. Intell. 36(9): 1847-1859, 2014.
[3] Senoussaoui, M., Kenny, P., Stafylakis, T., and Dumouchel, P., "A study of the cosine distance-based mean shift for telephone speech diarization", IEEE Trans. Audio, Speech Language Process. 22(1): 217 227, 2014.

[4] Daugman, J., "How iris recognition works?", IEEE Trans. Circuits Syst. Video Technol. 14(1): 21-30, 2004.

[5] M. R. RAJPUT, and M. WAGHMARE, "IRIS FEATURE EXTRACTION AND RECOGNITION BASED ON DIFFERENT TRANSFORMS", International Journal of Advances in Science Engineering and Technology, ISSN: 2321-9009 Volume1, Issue- 1, 2013.

[6] Kyaw, K., S., S., "Iris recognition system using statistical features for biometric identification”, In: Proceedings of international conference on electronic computer technology. pp. 554-556, 2009.

[7] R., M., Haralick, K., Shanmugam, and I. Dinstein Textural Features for Image Classification. IEEE Trans. on Systems, Man and Cybernetics (1973)610 - 621.

[8] B. Pathak, and D. Barooah "TEXTURE ANALYSIS BASED ON THE GRAY-LEVEL CO-OCCURRENCE MATRIX CONSIDERING POSSIBLE ORIENTATIONS", Biswajit P. and Debajyoti B., Vol. 2, Issue 9, 2013

[9] CASIA-Database. http://www.cbsr.ia.ac.cn/english/IrisDatabase.asp.

[10] Jyoti., M., and Dhiraj, G., "Reference Threshold Calculation for Biometric Authentication", I. J. Image, Graphics and Signal processing, 2,46-53, 2014.

[11] R. P. Wildes, 1996 Automated Noninvasive Iris Recognition System and Method", United States Patent, no. 5572596

[12] Charles, O., U., S., S., D., and W. Woo, "Iris feature extraction using principally rotated complex wavelet filter". IEEE International Conference on Computer Vision and Image Analysis Applications, Print ISBN: 978-1-47997185-5, 2015.

[13] Ankush K., "Development of novel feature for iris biometric", Department of Computer Science and Engineering, National Institute of Technology Rourkela 2013 